\title{
GNSS: status, modelagem atmosférica e métodos de posicionamento
}

\author{
GNSS: status, atmospheric modeling and positioning methods
}

\author{
Daniele Barroca Marra Alves ${ }^{1}$ \\ Pedro Augusto Giraldes de Abreu ${ }^{1}$ \\ Jéssica Saldanha Souza ${ }^{1}$
}
${ }^{1}$ FCT/UNESP - Campus de Presidente Prudente - Departamento de Cartografia. CEP 19060-900, Presidente Prudente, SP, Brasil.
danibarroca@fct.unesp.br,pe.abreu@hotmail.com,jessicasaldanha.souza@hotmail.com

\begin{abstract}
RESUMO - O GNSS (Global Navigation Satellite System) é cada vez mais empregado para realizar posicionamento. Além da modernização dos sistemas já existentes, como no caso do GPS (Global Positioning System) e GLONASS (Global'naya Navigatsionnaya Sputnikovaya Sistema), também existem novas iniciativas se estabelecendo, como o GALILEO e mais recentemente o Beidou/Compass. O objetivo desse artigo é apresentar o "estado da arte" desses sistemas, o status em que se encontram. Além disso, discorrer sobre os efeitos atmosféricos que influenciam na propagação dos sinais GNSS e consequentemente no posicionamento. Assunto de grande relevância, visto que o Brasil possui uma localização geográfica que prioriza tais efeitos, principalmente no que diz respeito aos efeitos ionosféricos. Por fim, descrever e apresentar resultados de diferentes métodos de posicionamento GNSS.
\end{abstract}

Palavras-chave: GNSS, Efeitos Atmosféricos, Posicionamento.

\begin{abstract}
GNSS has been widely used to realize positioning. Besides modernization of existent systems, like GPS (Global Positioning System) and GLONASS (Global'naya Navigatsionnaya Sputnikovaya Sistema), there are new initiatives, like GALILEO and Beidou/Compass. The purpose of this paper is present the "state of the art" of these systems. Besides, write about atmospheric effects that affect the GNSS signals propagation and consequently the positioning. This theme has great interest, Brazil has its territory in a privileged location, mainly concerning ionospheric effects. Finally, describe and present some results of GNSS positioning methods.
\end{abstract}

Keywords: GNSS, Atmospheric Effects, Positioning.

\section{INTRODUÇÃO}

Nos últimos anos tem sido cada vez maior o interesse em se realizar o posicionamento de feições terrestres com alta acurácia (erro de poucos centímetros). Nesse sentido, as tecnologias espaciais vêm sendo amplamente empregadas. O GNSS (Global Navigation Satellite System), uma das tecnologias espaciais de posicionamento mais avançadas que surgiu recentemente, tem revolucionado as atividades relacionadas com posicionamento.

O GNSS desperta interesse da comunidade em geral, e seu pleno domínio é essencial para que se faça bom uso do sistema. O número de aplicações é muito amplo e continua aumentando, indo desde a Geodésia, a Geodinâmica, a Agricultura de Precisão, a Meteorologia, a Aeronomia, a Navegação, até as atividades de lazer. Há uma expectativa de que em breve a maioria dos dispositivos móveis (veículos, celulares, colhedoras, máquinas, etc.) terá um receptor de posicionamento por satélite instalado a bordo. Trata-se, portanto, de uma tecnologia indispensável para várias áreas do conhecimento (transporte, exploração de óleo e gás, mapeamento, sistemas de informação geográfica, etc.).
Com o objetivo de usufruir do potencial dos sistemas de posicionamento global existentes, com o passar dos anos, os métodos de posicionamento vêm se aprimorando. Além disso, novas técnicas têm surgido com o objetivo de realizar posicionamento com alta acurácia. Nesse sentido, uma grande tendência tem sido o uso de dados de redes de estações de referência. O posicionamento realizado utilizando dados de redes tem se tornado mais efetivo nos últimos anos, devido, principalmente, a alta acurácia proporcionada pelo método denominado RTK (Real Time Kinematic) em rede. Detalhes sobre o RTK em rede podem ser obtidos em Alves $(2008 ;$ 2011) e Alves e Monico (2011). Além do RTK em rede, um método que vem sendo muito discutido é o PPP em tempo real, que para alcançar alta acurácia também emprega dados de redes, tanto na estimativa do erro do relógio do satélite (ZHANG et al., 2011; MARQUES, 2012) como nos métodos de solução das ambiguidades (GE et al., 2008; HAUSCHILD, 2010). Uma outra possibilidade, para aplicações que requerem acurácia decimétrica, é o DGPS em rede (ALVES et al., 2011).

Mas, para que os métodos de posicionamento atinjam a acurácia pretendida, é indispensável realizar uma modelagem atmosférica (ionosfera e troposfera) 
adequada. No que concerne à troposfera, embora existam os modelos empíricos (por exemplo Saastamoinen e Hopfield) eles podem não ser apropriados para a realidade brasileira, principalmente quando resultados altamente acurados são requeridos. Para minimizar essa limitação, surgem os modelos de PNT (Previsão Numérica do Tempo) (SAPUCCI et al., 2008). Outro fator extremamente importante para o posicionamento são os erros que ocorrem devido à ionosfera. A refração ionosférica é uma das maiores fontes de erro no posicionamento GNSS. O Brasil, por ter grande parte do seu território no equador geomagnético é afetado de forma intensa pela ionosfera (MATSUOKA, 2007; AGUIAR, 2010). Nesse artigo, o objetivo principal é apresentar o status atual dos GNSS, bem como a problemática envolvida na modelagem atmosférica e resultados no posicionamento GNSS.

\section{GNSS}

Os principais sistemas globais que compõem o GNSS são: GPS (Global Positioning System), GLONASS (Global'naya Navigatsionnaya Sputnikovaya Sistema), Galileo e mais recentemente o Beidou/Compass.

O GPS, desenvolvido pelos Estados Unidos, se encontra em plena operação e em fase de modernização. Em setembro de 2005 foi lançado o primeiro satélite GPS modernizado, hoje (novembro de 2012) já se tem 10 desses satélites em operação (7 do bloco IIRM e 3 do bloco IIF) (www.navcen.uscg.gov). O último lançamento (satélite do bloco IIF) foi realizado em outubro de 2012. Os satélites do bloco IIRM possuem o novo código civil na portadora L2 denominado L2C. Já os satélites do bloco IIF, além do L2C, também transmitem sinais em uma nova portadora, a L5 (www.gps.gov). Atualmente, a constelação GPS conta com 31 satélites operacionais.

O GLONASS, similar ao GPS, desenvolvido na antiga URSS, é hoje de responsabilidade da República da Rússia. Foi declarado operacional em 1995 (http://www.nis-

glonass.ru/en/glonass/development_history/), mas não teve a devida manutenção por alguns anos. Atualmente encontra-se com a constelação completa (31 satélites). Em 14/11/2012 apenas 24 satélites estavam em operação (http://www.glonass-ianc.rsa.ru/). Geralmente, o número de satélites em operação na constelação GLONASS varia entre 22 e 24.

No que concerne ao Galileo, sistema europeu, foram lançados dois satélites em outubro de 2011 e mais dois em outubro de 2012 (http://www.esa.int/). Já haviam sido lançados previamente para testes outros dois satélites: em 2005 (GIOVE-A) e em 2008 (GIOVE-B). Espera-se que o sistema esteja com capacidade operacional inicial por volta de 2014 com 18 satélites em órbita. Já a constelação completa é esperada para 2020 (http://www.galileoic.org).

A China, que no passado revolucionou a navegação a partir do desenvolvimento da bússola, surpreendeu com o Beidou/Compass. A ideia do sistema começou como um serviço regional e mais tarde expandiu para um serviço global. Atualmente, o sistema conta com 16 satélites, destes 5 satélites são MEO (Medium Earth Orbit), similares ao GPS. Os demais satélites são de órbita geoestacionária ou geoestacionária inclinada (IGSO Inclined Geostationary Orbit) (http://www.insidegnss.com/node/3246). O último lançamento de satélites MEO foi realizado em setembro de 2012 (satélites M5 e M6). Espera-se que a constelação esteja completa em 2020. A página oficial do Beidou/Compass é www.beidou.gov.cn.

Além desses sistemas, compõem o GNSS, os chamados SBAS (Satellite Based Augmentation System), tais como o WAAS (Wide Area Augmentation System) nos Estados Unidos, o EGNOS (European Geostationary Navigation Overlay Service) na Europa, o MSAS (Multifunctional Satellite Augmentation System) no Japão, o GAGAN (GPS Aided GEO Augmented Navigation) na Índia, além de outras iniciativas similares. Assim, além de alta acurácia, o GNSS pode proporcionar integridade e disponibilidade do sistema (SEEBER, 2003).

Espera-se que num futuro próximo, com a existência de diversos sistemas de posicionamento por satélite disponíveis, seja realizada efetivamente a integração entre tais sistemas. Com isso, poderá ser obtido um posicionamento mais acurado, devido principalmente a grande quantidade de dados. Schönemann et al. (2011) discute esse tema.

\section{EFEITOS ATMOSFÉRICOS}

Para fins de posicionamento geodésico a atmosfera terrestre pode ser dividida em troposfera e ionosfera. Cada camada com características e efeitos nos sinais GNSS bem distintos.

\subsection{Troposfera}

A Troposfera é considerada a camada mais superficial da atmosfera terrestre, com espessura média de aproximadamente $50 \mathrm{~km}$. Ela é um meio não dispersivo para a faixa de frequência do GNSS. O atraso troposférico pode ser dividido em duas componentes: hidrostática e úmida. A primeira representa cerca de $90 \%$ do atraso, é gerada pela influência da atmosfera hidrostática (ZHD Zenithal Hydrostatic Delay). O erro devido a essa componente é de aproximadamente $2,3 \mathrm{~m}$ no zênite. O ZHD pode ser determinado com razoável precisão, pois sua variação é pequena, o que o torna de fácil modelagem. A segunda componente ocorre devido à atmosfera úmida (ZWD - Zenithal Wet Delay), ou seja, pela influência do vapor d'água atmosférico. Tal atraso é geralmente menor, representando cerca de $10 \%$ do atraso troposférico total. Porém, sua variação temporal e espacial é muito maior, chegando a $20 \%$ em poucas horas, o que torna impossível uma previsão adequada a partir de medidas da umidade na superfície (SPILKER, 1994). O atraso troposférico total (ZTD - Zenithal Tropospheric Delay) pode aumentar cerca de 10 vezes próximo ao horizonte $\left(10^{\circ}\right.$ de elevação) (SEEBER, 2003)

No posicionamento geodésico, devido à variabilidade e difícil modelagem, a variável que merece 
uma maior atenção nos efeitos troposféricos é o ZWD. Na Fig. 1 são apresentados os valores do ZWD estimados para a estação PPTE da RBMC (rede Brasileira de Monitoramento Contínuo) no ano de 2011. Os valores do ZWD foram calculados a cada $5 \mathrm{~min}$ utilizando o software Gipsy Oasis disponível na FCT/UNESP. É possível avaliar na figura que, como esperado, os maiores e menores índices de umidade são obtidos no verão e inverno respectivamente.

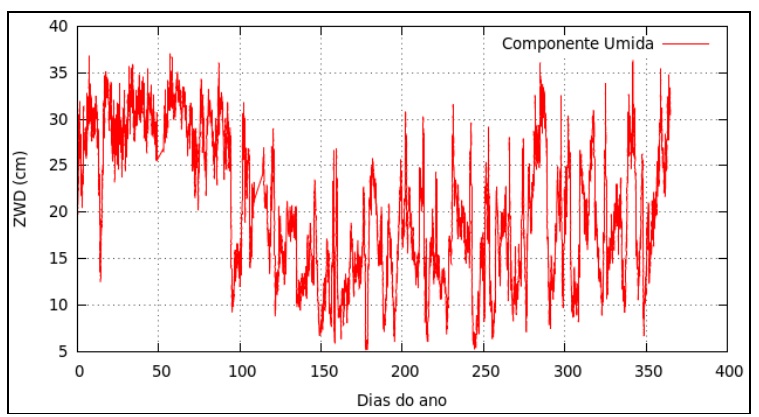

Figura 1 - ZWD do ano de 2011 para a estação PPTE obtido com dados GNSS a partir do processamento no software GIPSY.

Ao invés de estimar o ZWD, outra possibilidade é utilizar modelos. Quando se emprega modelos empíricos (Hopfield ou Saastamoinen por exemplo), o valor do ZWD obtido é constante (Fig. 2), independente da hora do dia, dia do ano ou até mesmo da localização geográfica. Saastamoinen utiliza apenas o módulo do valor da latitude e a altura da estação, enquanto Hopfield não usa esse tipo de informação. Já quando modelos de PNT são utilizados, o ZWD e o ZHD são calculados especificamente para cada dia e localização geográfica, além de possuírem variação no decorrer do dia (Fig. 2).

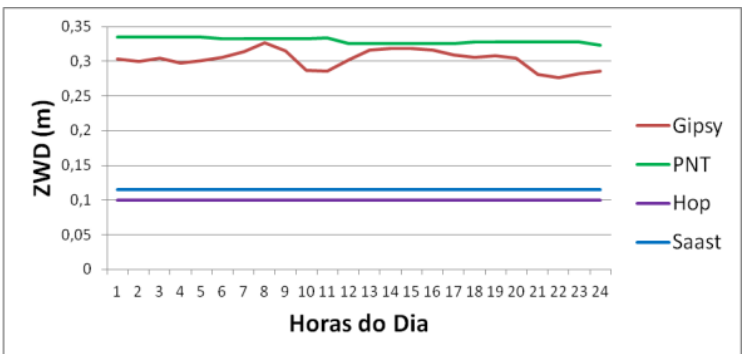

Figura 2 - ZWD da estação PPTE para o dia 08/01/2011 obtidos com dados GNSS a partir de processamento no software GIPSY e os modelos de PNT (Previsão Numérica do Tempo) do CPTEC/INPE, Saastamoinem e Hopfield.

O uso de modelos de PNT (Previsão Numérica do Tempo), ao invés de modelos empíricos, tem sido amplamente utilizado e indicado pela comunidade científica (BOEHM et al., 2006). No Brasil, o CPTEC/INPE possui o único modelo global de PNT em modo operacional da América Latina. A formulação matemática do modelo pode ser encontrada em Sapucci et al. (2008), em Alves (2008) ou na página do CPTEC/INPE (http://satelite.cptec.inpe.br/zenital/). Testes iniciais foram realizados em Alves et al. (2006) e o modelo de PNT apresentou melhores resultados que o modelo empírico de Hopfield no posicionamento em redes, melhorias de até $19 \%$ foram obtidas.

\subsection{Ionosfera}

A ionosfera é uma das principais fontes de erros no posicionamento GNSS. A radiação solar causa a fotoionização da atmosfera terrestre nas altas altitudes, criando, na atmosfera superior, regiões parcialmente ionizadas, conhecidas como ionosfera, que estão compreendidas, aproximadamente, entre 50 a $1000 \mathrm{~km}$ de altura. A ionosfera, como um meio dispersivo para a faixa de frequência GNSS, afeta a modulação e a fase da portadora, fazendo com que sofram, respectivamente, um retardo e um avanço (CAMARGO, 1999).

$O$ efeito da refração ionosférica depende da frequência usada e, consequentemente, do índice de refração. Alem disso, é proporcional ao Conteúdo Total de Elétrons (Total Electron Contents - TEC), ou seja, o número de elétrons presentes ao longo do caminho percorrido pelo sinal entre o satélite e o receptor, e inversamente proporcional ao quadrado da frequência. Outros parâmetros que influenciam a refração ionosférica são principalmente a atividade solar e o campo geomagnético. A refração ionosférica também depende da localização geográfica e do tempo (SEEBER, 2003). Assim, o TEC apresenta variações diárias (Fig. 3), sazonais, geográficas e de longo período (ciclo solar de 11 anos).

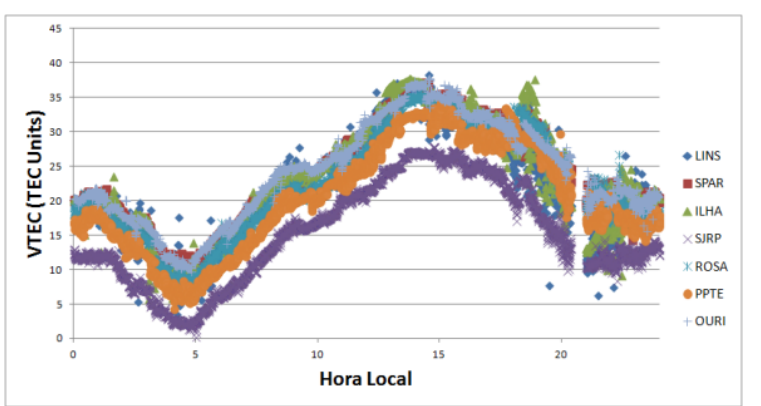

Figura 3 - VTEC (Vertical TEC) obtido a partir de dados GNSS de 7 estações da Rede GNSS/SP para o dia 16/10/2009 (24h de dados).

Além dos efeitos já citados, comparecem irregularidades ionosféricas, como à anomalia equatorial, tempestades geomagnéticas, bolhas ionosféricas e cintilação. Efeitos que devem se intensificar no próximo ano devido ao pico da atividade solar que deverá ocorrer em 2013 (http://solarscience.msfc.nasa.gov/predict.shtml).

Algo que tem recebido bastante atenção da comunidade científica é referente à cintilação ionosférica. As cintilações ionosféricas são mudanças rápidas que ocorrem na fase e amplitude do sinal de rádio recebido, as quais são causadas por irregularidades na densidade de elétrons ao longo do caminho percorrido pelo sinal na ionosfera, podendo enfraquecer o sinal recebido pelos receptores GNSS, fazendo que ocorra em muitos casos a degradação ou até mesmo perca do sinal (CONKER et al., 2003). 
O Brasil, que possui grande parte de seu território situado no equador geomagnético, sofre grande influência da cintilação ionosférica. A Fig. 4 apresenta os valores do índice $\mathrm{S} 4^{1}$ considerando o TU (tempo universal), para um dia considerado menos (02/07/2012) e mais intenso (20/10/2012). Foram empregados dados da estação PRU1 localizada na cidade de Presidente Prudente, SP. Quanto maior o índice $\mathrm{S} 4$, maior é o efeito da cintilação ionosférica. Note que para julho a cintilação ionosférica é mais branda, chega no máximo próximo a 0,8 . Já para outubro os valores de S4 se aproximam de 1,8, principalmente nos horários após o por do sol.

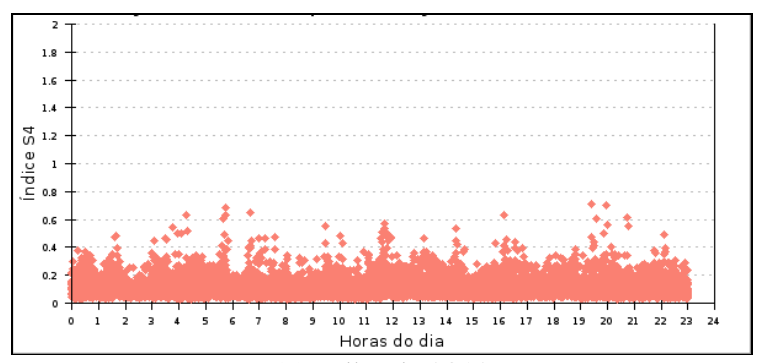

(a) Julho de 2012

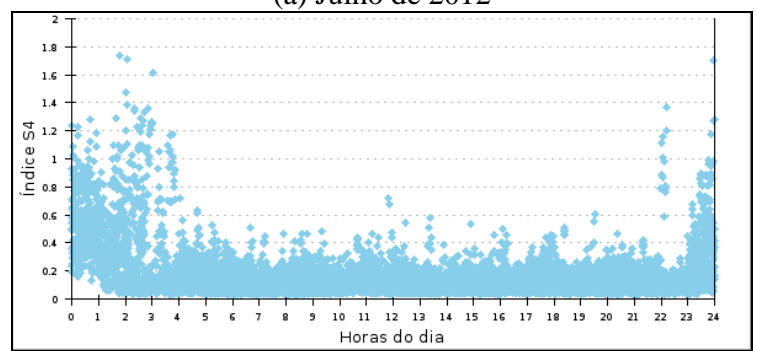

(b) Outubro de 2012

Figura 4 - Índice S4 de cintilação ionosférica para um dia considerado de cintilação branda (a) e intensa (b)

Devido a todos esses pontos, a ionosfera tem sido um dos maiores obstáculos quando se deseja prover posicionamento com acurácia centimétrica, ou mesmo decimétrica. No Brasil, onde as condições ionosféricas são muito variáveis, existe a necessidade de desenvolver modelos robustos que sejam adequados a essa realidade. Uma possibilidade é a utilização da modelagem regional da ionosfera (CAMARGO, 1999; ALVES, 2008; 2011). Algo que também vem sendo muito explorado pela comunidade científica é a ideia de grade ionosférica (GI) (AGUIAR, 2010). Mas, também comparecem outros métodos como a modelagem tomográfica por exemplo. Alves (2008) apresenta os principais modelos empregados pela comunidade científica.

\section{POSICIONAMENTO GNSS}

O GNSS tem sido cada vez mais utilizado para realizar posicionamento. Empregando essa tecnologia pode-se realizar o PP (posicionamento por ponto), PPP (posicionamento por ponto preciso), DGPS (GPS

${ }^{1}$ Segundo Conker et al. (2003) quando atingi-se o índice S4 > 0,707, alguns modelos de receptores são considerados bloqueados pela cintilação, ou seja, perdem quase ou totalmente o sinal transmitido. diferencial), relativo ou posicionamento baseado em redes. Métodos que possuem características distintas (SEEBER, 2003) com suas respectivas vantagens e desvantagens.

No PP ou PPP o conceito básico é a utilização de apenas um receptor para realizar o posicionamento. Esses métodos se diferenciam pelas observáveis empregadas e a acurácia alcançada. Enquanto no PP a acurácia gira em torno de metros, o PPP pode alcançar acurácia centimétrica. Hoje, o PPP em tempo real representa o estado da arte no posicionamento por ponto. Para mais detalhes consulte, por exemplo, Zhang et al. (2011) e Marques (2012).

Um método bastante empregado é o DGPS, que pode proporcionar acurácia métrica (SEEBER, 2003), ou até mesmo submétrica. Mas, devido à decorrelação espacial dos erros, a distância entre a base e o usuário é limitada. Para sanar essa limitação, outra possibilidade é o DGPS em rede (ALVES et al., 2011). A Fig. 5 apresenta a melhoria do DGPS em Rede em relação ao DGPS para 3 dias de processamento. Na média, o EMQ para o DGPS em rede foi de 31,65 e $63,5 \mathrm{~cm}$ nas componentes horizontal e vertical respectivamente. Já para o DGPS, o EMQ foi de aproximadamente 69,3 e $87,5 \mathrm{~cm}$ nas mesmas componentes.

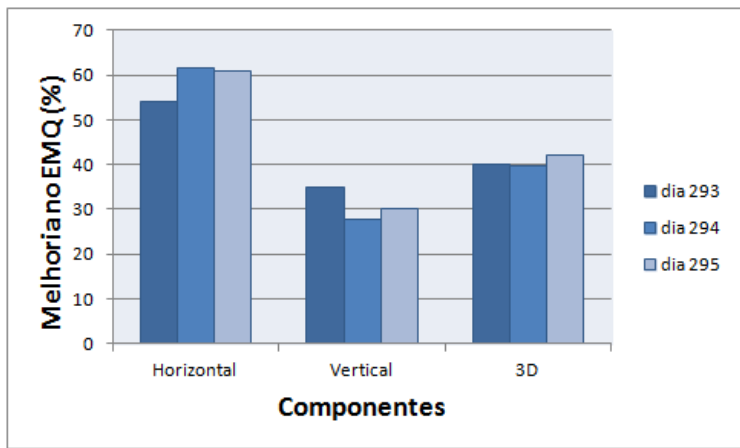

Figura 5 - Melhoria do EMQ do DGPS em rede em relação ao DGPS.

Com o DGPS em rede, a acurácia obtida é superior ao DGPS, como visto na figura, mas devido a observável empregada, pseudodistância, espera-se alcançar no máximo acurácia decimétrica com esse tipo de posicionamento (como visto nos testes). Algo que é suficiente para inúmeras aplicações. Além disso, com a modernização do GPS e os novos sistemas, provavelmente essa acurácia obtida com pseudodistância deve ser melhorada.

Dentre os métodos de posicionamento existentes, um dos mais empregados é o relativo. Além da alta acurácia proporcionada, algo que tem fortalecido o método é a disponibilização de dados das redes ativas. Utilizando dados dessas redes para a estação base, o usuário pode realizar o posicionamento relativo com apenas um receptor. No posicionamento relativo, um método bastante empregado é o RTK, devido à alta produtividade e a acurácia centimétrica proporcionada. Mas, para que isso ocorra, o comprimento das linhas base deve ser curto, dependendo principalmente das condições ionosféricas. 
Para sanar essa limitação surge o posicionamento baseado em redes, ou simplesmente, RTK em rede. A Fig. 6 ilustra a acurácia obtida na componente vertical com o método relativo (menor linha base na rede) e o posicionamento em rede para a rede GNSS/SP. A melhoria obtida pelo uso do posicionamento em rede foi em torno de $50 \%$ em altimetria. Para a planimetria essa melhoria foi de $37 \%$.

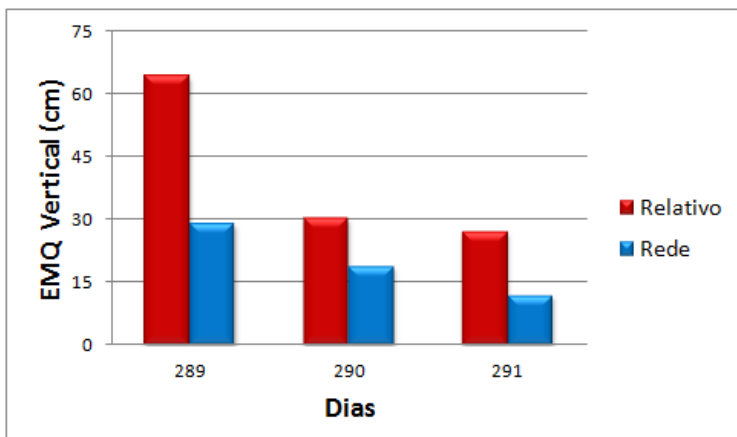

Figura 6 - EMQ obtido empregando o posicionamento relativo e o posicionamento baseado em redes utilizando dados da Rede GNSS/SP.

\section{CONCLUSÕES}

Cada vez mais o GNSS tem se tornado uma ferramenta indispensável para realizar posicionamento com alta acurácia. A modernização dos sistemas, como do GPS e GLONASS, e o surgimento de novas iniciativas, como o GALILEO e o BEIDOU/COMPASS, tem contribuído muito nesse sentido.

Algo que também deve ser destacado é o aprimoramento dos métodos de posicionamento, além do surgimento de novas técnicas. Existe uma grande variedade de métodos, que devem ser empregados de acordo com a acurácia almejada para uma determinada aplicação.

A expectativa é que num futuro próximo, com a existência de diversos sistemas de posicionamento por satélites disponíveis, seja realizada a integração entre tais sistemas. Com essa integração, e o avanço dos métodos de posicionamento, a tendência é obter alta acurácia instantaneamente, em campo, com uma facilidade muito maior.

\section{AGRADECIMENTOS}

A primeira autora agradece ao CNPQ (Conselho Nacional de Desenvolvimento Científico e Tecnológico) pelo financiamento da bolsa de Produtividade em Pesquisa.

\section{REFERÊNCIAS}

AGUIAR, C. R. Grade Ionosférica para Aplicações em Posicionamento e Navegação com GNSS. 2010. $258 \mathrm{f}$. Tese (Doutorado em Ciências Cartográficas) Universidade Estadual Paulista, Presidente Prudente.

ALVES, D. B. M.; MONICO, J. F. G.; DALBELO, L. F. A.; SAPUCCI, L. F.; CAMARGO, P. O. VRS Concept Using NWP and Mod_Ion_FK: Preliminary
Results in Brazil. In: International FIG Congress, Munique, Alemanha. Proceedings..., 2006.

ALVES, D. B. M. Posicionamento Baseado em redes GPS utilizando o conceito de estação virtual, 2008. Tese (Doutorado em Ciências Cartográficas) Universidade Estadual Paulista, Presidente Prudente.

ALVES, D. B. M. Desenvolvimento e Implantação do RTK em Rede para Posicionamento Geodésico no Estado de São Paulo, 2011. Pós-Doutorado Universidade Estadual Paulista - Departamento de Cartografia, Presidente Prudente.

ALVES, D. B. M.; MONICO, J. F. G. GPS/VRS positioning using atmospheric modeling. GPS Solutions (Heidelberg), v.15, n.3, DOI 10.1007/s10291-010-0187-3, p. 253-261, 2011.

ALVES, D. B. M.; DALBELO, L. F. A.; MONICO, J. F. G.; SHIMABUKURO, M. H. First Brazilian Real Time Network DGPS through the Internet: Development, Application and Availability Analyses. Journal of Geodetic Science. v.1, p. 1-7, 2011.

BOEHM, J.; NIELL, A.; TREGONING, P.; SCHUH, H. Global Mapping Function (GMF): A New Empirical Mapping Function Based on Numerical Weather Model Data. Geophysical Research Letters. v.33. 2006.

CAMARGO, P. O. Modelo Regional da Ionosfera para Uso em Posicionamento com Receptores GPS de Uma Freqüência. 1999. 191f. Tese (Doutorado em Ciências Geodésicas) - Universidade Federal do Paraná, Curitiba.

CONKER, R. S.; EL-ARINI, M. B.; HEGARTY, C. J.; HSIAO, T. Modeling the Effects of Ionospheric Scintillation on GPS/Satellite-Based Augmentation System Availability, Radio Science, Vol. 38, 2003.

GE, M.; GENDT, G.; ROTHACHER, M.; SHI, C.; LIU, J. Resolution of GPS Carrier-Phase Ambiguities in Precise Point Positioning (PPP) with Daily Observations. Journal of Geodesy, v.82, n.7, DOI 10.1007/s00190-007-0187-4, p.389-399. 2008.

HAUSCHILD, A. Precise GNSS Clock-Estimation for Real Time Navigation and Precise Point Positioning, 2010. PhD Thesis. Technical University of Munich, Monique, Alemanha.

MARQUES, H. A. M. PPP em Tempo Real com Estimativa das Correções dos Relógios dos Satélites no Contexto de Rede GNSS. 2012. Tese (Doutorado em Ciências Cartográficas) - Universidade Estadual Paulista, Presidente Prudente.

MATSUOKA, M. T. Influência de Diferentes Condições da Ionosfera no Posicionamento por Ponto com GPS: Avaliação na região Brasileira. 2007. Tese (Doutorado em Ciências Cartográficas) Universidade Estadual Paulista, Presidente Prudente.

SAPUCCI, L. F.; MONICO, J. F. G.; MACHADO, L. A. T.; ROSA, G. P. S. Avaliação das Previsões do Atraso Zenital Troposférico para a América do Sul obtidas usando Modelo de Previsão Numérica de Tempo com Alta Resolução Espacial. Boletim de Ciências Geodésicas. v.14, n.4, 2008. 
SCHÖNEMANN, E.; MATTHIAS, B.; SPRINGER, T. A new Approach for GNSS Analysis in a Multi-GNSS and Multi-Signal Environment. Journal of Geodetic Science. v.1, n.3, p. 204-214, 2011.

SEEBER, G. Satellite Geodesy: Foundations, Methods, and Applications. Berlin, New York: Walter de Gruyter, 2003.

SPILKER, J., J. JR., Tropospheric Effects on GPS. American Institute of Aeronautics and Astronautics. Vol 1 Capítulo 13, p. 517 - 546, 1994.

ZHANG, X.; LI, X.; GUO, F. Satellite Clock Estimation at $1 \mathrm{~Hz}$ for Realtime Kinematic PPP Applications. GPS Solutions. V.15, p.315-324, 2011. 